\title{
Function abstraction in automatic digital-circuit design
}

\author{
J.-G. Wu \\ Y.H. Hu, PhD \\ W.P.-C. Ho, PhD \\ Prof. D.Y.Y. Yun, PhD
}

\begin{abstract}
In the paper, a novel approach for automating the functional design of digital circuits is presented. The goal of digital-circuit design is to generate a workable and efficient design from high-level specifications. Function abstraction is an important step, whereas the specification of the target circuit is purely behavioural. It finds the essential functions of the desired behaviour. These functions can then be easily bound to physical modules. The design space for a behavioural description is huge. Therefore, it is important to have a powerful search strategy which will lead to a near optimum solution in reasonable time. We use the heuristic best-first search and metaplanning techniques to control the decision making and optimise the final design. Experimentals results are presented to compare the effectiveness of these search strategies.
\end{abstract}

\section{Introduction}

Digital systems design is a process which transforms high-level behavioural specification into physical circuits. This process is largely an optimisation problem, i.e. a problem of mediating between minimising the use of limited resources (e.g. physical size, cost, power consumption) and maximising performance (e.g. speed, functionalities). To reduce the design complexity and cost, and to improve the reliability and testability of the circuit, it is vital to arrive at a design which uses a minimum number of logic elements.

Existing logic minimisation systems often focus on combinational logic minimisation [1-5] or on local minimisation of function modules coming from the structural information in system specifications [6-12]. Recently, we have proposed a model-based method for designing a

Paper $6826 \mathrm{G}$ (E10), first received 11 th July 1988 and in revised form 12th May 1989

J.G. Wu is with the Department of Electrical Engineering, National Taiwan University, PO Box 23-5, Taipei, Taiwan 10098, Republic of China

Y.H. Hu is with the Department of Electrical and Computer Engineering. University of Wisconsin-Madison, 1415 Johnson Drive, Madison, WI 53706 , USA

W.P.C. Ho is with the Department of Electrical Engineering and Systems, University of Southern California, LA 90089-0781, USA

Prof D.Y. Yun is with the Department of Computer Science and Engineering, Southern Methodist University, 306 Science Information Center, Dallas, Texas 75275 , USA digital circuit from purely behavioural specifications [13]. This paper will focus on a function minimisation problem which arises in the digital system design process. We assume that in the specification of a digital system, there are a mixture of functional and behavioural descriptions for different parts of the system. The functional parts explicitly indicate the functional modules used and the functions to be performed. Thus, only local minimisation can be done on the function modules to eliminate redundancy and perhaps, to a limited extent, improve performance. On the other hand, the behavioural parts in the specification characterise only the input/output timing relationships. Since they are not bound to any specific function modules in the specifications, it presents a great opportunity for the exploration of globally optimised structural designs.

In our research work, the behavioural description which describes the timing relationship among interface signals (like timing diagrams) is characterised by seqquences of primitive operations. A primitive operation, which a low-level bit-wise and time-instant function of digital circuits, can be covered by some higher level functions which cover other primitive operations as well. In other words, a function is a higher level behavioural identity which 'abstracts' the behaviour of a sequence of primitive operations. This process is called function abstraction. A function module is a structural entity which physically implements one or more functions. Based on these definitions, the digital systems design problem can then be formulated into two subsequent optimisation problems:

(i) the selection of a minimum set of functions to cover the given sequence of primitive operations

(ii) the selection of a minimum set of function modules to implement these chosen functions.

Our approach is unique in that other approaches try to find function modules from (behavioural) specifications directly, whereas we first compile the specifcations into low-level primitive operations, which admit the subsequent two-stage optimisation, before mapping into function modules. We believe this approach is more promising in that it provides more opportunity and flexibility for design improvement.

We formulate the process of function abstraction from primitive operations as a constructive search problem, in which many possible functions can cover some part of the primitive operations in the specifications. The problem of how to select a minimum set of functions having the desired behaviour, and how to do so in reasonable amount of time, is the focus of this paper. 
There are several formal methods to perform combinational logic minimisation, such as in References 1 and 2. Technology-independent (logically but not physically minimised) implementations can be generated by these methods. Some systems [3-5] use heuristics to perform local minimisation and implementation of Boolean equations. These systems deal only with combinational circuits. The minimisation of combinational logic may solve part of the minimisation problem. but in a digital system, sequential circuits are also important parts. Moreover, combinations of combinational circuits and sequential circuits may sometimes be optimised together, so that combinational and sequential circuits should be considered together in minimisation to get better results.

Most of the existing knowledge-based digital circuit design systems, such as DAA $[6,7]$, ADAM $[8,9]$ and VEXED [10], use top-down strategy as the framework of design. Under this framework, the input design specifications are refined hierarchically into submodules until primitive modules are reached. Specification decomposition is done using structural information present in the design specifications. In this top-down design technique imprecise cost estimates are made as the final design is not yet available. You are more likely to get a workable design than an optimised design.

DAA (Design Automation Assistant) is a rule-based system. It decomposes the process of a digital system design into four subtasks. The first subtask, global allocation, identifies and allocates the needed global objects, i.e. storage elements, contraints and controller. The second subtask, value trace allocation, partitions the whole design into smaller blocks and allocates their internal clock phases, operators, registers and controller. The third step, SCS (a Structure and Control Specification language) allocation, examines and performs possible local improvements. The last subtask, global improvements, removes unneeded modules and tries a better sharing of modules.

The two minimisation steps in DAA are based on rules and work on structural function modules bound to func tions in the system specification. A rule sees only a small part of the entire circuit. Thus only local minimisation can be made. DAA has no backtracking. It needs a huge set of rules to find good designs as opposed to workable designs. BUD [14], which is the preprocessor of DAA uses a bottom-up framework to analyse the target design globally. Clustering is made in BUD which gives bette information of the layout of the final design, but it is still doing local minimisation on structure.

VEXED is a knowledge-based design system. Knowledge about decomposition and implementation methods of functions are used to guide the planning of the design process. Constraints are propagated to assure that all interactions among subproblems are considered. Since the lack of control knowledge, VEXED can only list al possible decompositions of the specification of a module and the user decides which decomposition is made. It is only a design support system.

In the proposed design paradigm of Reference 11 , there are several experts at each level of abstraction to do the tasks of constraints propagation, planning, refinement, optimisation and evaluation. The planner selects a most promising straegy or style for specifications refinement. The refiner transforms the desired behavioural specifications into lower level design abstraction. The

optimiser tries to improve the design without degrading its performance. The evaluator estimates the performance of design and propagates constraints upward for relaxation of constraints or downward for further refinement

The goal of ADAM is to manage a number of design automation programs into a single framework. Planning techniques are used to build an abstract design plan which contains a possible sequence of design activities (the use of design tools) [9]. Execution of the design plan is performed after estimation and after finding the feasibility of the design activities.

There is only one other system, Synapse [12], which uses the primitive logic description. At each level of abstraction, a set of algebraic primitive operations is used to model the behaviour of the target circuit. The system goal is to map very high level specification of a problem into a custom VLSI circuit. The global synthesis strategy of this system is the same as other expert systems. First, the input expression is simplified to lower expressions. Then, the expressions are factored to obtain an architecture. Finally, the architecture is converted to a layout. The specification is expressed in algebraic form such that axioms and rewriting rules can be used to simplify the input expression. Since that system uses a theoremproving technique which is devoid of planning knowledge and heuristics, there is no indication that it can solve practical problems due to a combinational explosion.

Our approach, compared with other existing knowledge-based digital circuit synthesis systems, is distinctive in the following aspects:

(i) Most systems represent the design knowledge by ad hoc rules which often focus on local features and may be limited to make only local optimisation. On the other hand, we use a formal heuristic search method to perform optimisation. Because global features can be formulated into the cost function, this approach has better potential to achieve global optimisation

(ii) In a rule-based system, implicit knowledge may be redundantly encoded in several rules and hence a huge set of rules may be needed to accomplish a 'good' design. In our design system, fundamental theorems of digital circuits and behaviour of logic functions and modules are explicitly expressed so that the problem of redundancy and inconsistency may be largely eliminated

(iii) Most existing systems use top-down design strategy in which functional blocks in the target system's description may restrict the structure of the final design. In our case, a bottom-up design strategy is subscribed. Essential functions are abstracted from a set of purely behavioural specifications. As such, there is no structural information to restrict the design options. On the other hand, top-down design strategy is efficient in large systems design, whereas the bottom-up strategy is more suitable for medium-sized circuits design.

\section{Model-based automatic digital circuit design} system

\subsection{Representation of functional/structural entities}

This Subsection defines the functional and structural entities related to the two minimisation subprocesses in our automatic design system.

3.1.1 Primitive operations: a set of five basic functional entities which represent the behaviour of the target system. They are [AND, OR, NOT, transmit and store]. This set of operations is complete in the sense that it can be used to describe the behaviour of all logic circuits

IEE PROCEEDINGS, VOL. 136, Pt. G, NO. 5, OCTOBER 1989 
including combinational, sequential and bus circuits. A primitive operation will be characterised using a regular expression with the following format:

operation(input ${ }_{1}$, input ${ }_{2}, \ldots$, control, output, timing)

The last three arguments of an operation are control signal, output and timing, respectively. The rest of the arguments are input signals. An empty argument implies that it is 'don't care' or unknown. [AND and OR] operations may have more than one input, whereas other operations have only one input. [Transmit and store] operations have a control signal. The timing of an operation may be a constant or a constant followed by an $r$. A constant timing means the operation is performed at that specific time instant. If the timing is a constant followed by an $r$, it means the operation is performed 'repeatedly' with the constant as its period.

Two declaration statements, value and input, are used to indicate the value of signals at one time instant. They have the format

declaration(signal, value, time)

This value will be referred to in the future. An input statement indicates the condition of an input signal which can be used as the control signal of other operations. A value statement keeps the value of a signal which will be used in future. It is used to link the information which has been processed at two or more different times.

3.1.2 A function: specifies the operations on the related signals in a time interval. During this interval, information is input, processed and then output. The relationship between the input information and output alone is sufficient to characterise a function. Intermediate operations are not important. For example, parallel-to-serial conversion is a function. It accepts certain input data at the beginning, and then sequentially sends the individual bits of those data as the output signal. What is important is the timing about the input and output of those data. How those data are stored and transferred during their operation is not our concern. Currently, we include simple functions such as and, or, not, set, reset, load, change states, count, parallel-to-serial conversion, serialto-parallel conversion, pattern generation, code conversion, control signal generation, and so on, in the prototype of a function abstraction program.

Most of the functions need control signals to guide their proper operation. For example, the function parallel-to-serial conversion needs a load data control signal to take in the parallel data. It also needs a clock signal to control the timing of all serial data output. These control signals are not included as part of the function. Thus, supporting primitive operations may be needed to generate the control signals. Since it is assumed that there is always a system clock signal, it needs no supporting operations to generate.

The behaviour of a function is represented by a set of primitive operations. For example, the behaviour of the parallel-to-serial conversion function, which transfers the $n$-bit data from its input $i_{1}-i_{n}$ to its one-bit output $a$ sequentially, includes the following primitive operations:

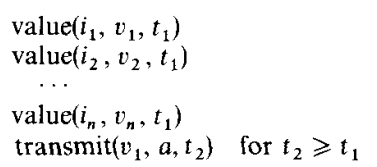

IEE PROCEEDINGS, Vol. 136, Pt. G, No. 5, OCTOBER 1989

$$
\begin{aligned}
& \operatorname{transmit}\left(v_{2}, a, t_{2}+1\right) \\
& \quad \cdots \\
& \operatorname{transmit}\left(v_{n}, a, t_{2}+n-1\right) .
\end{aligned}
$$

Its supporting operations are

value(ld, rising, $\left.t_{1}\right)$

and

value(ck, rising, $\left.t_{2}+i\right)$ for $i=0$ to $n-1$

3.1.3 Function modules: are physical entities that are used to implement logic functions. For example, AND gates, OR gates, inverters, latches, counters, decoders and read only memories (ROMs) are function modules. A function module can implement part of, one, or more than one function. For example, a latch can implement part of a change states function and a RS latch can implement functions set, reset and load. The behaviour of a function module is also represented by primitive operations [15]. Therefore, we can use the techniques of automated reasoning to resolve their relationship and examine whether a module can implement a function.

\subsection{Design subprocesses}

The process of a digital circuit design is decomposed into three subprocesses [13]. The system flow is shown in Fig. 1.

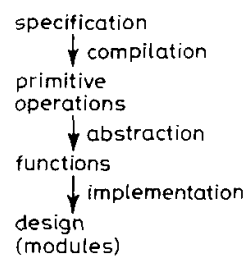

Fig. 1 System flow of automatic design system

The first subprocess, compilation, translates the purely behavioural description of a digital system given by the user into sequences of primitive operations. The description of a digital circuit is similar to the timing diagram. Only the relationship between input/output signals is described and no internal structure is shown. The primitive operations are used to specify the behaviour of digital circuits. The translation from a statement in the description to sequences of primitive operations is implemented as a one-to-one mapping in our system, although there may be other interpretations.

The second subprocess, abstraction, attempts to abstract and minimise a set of functions from the primitive operations from the compilation step. Primitive operations are grouped to form functions, and support operations are added to generate control signals and to fill in holes in the specification. This step tries to find a minimum number of functions to cover the desired behaviour of the target system. Maximum usage of the selected function, meaning that a minimum amount of unused operations are included, is also a part of the constraints in this step. The tasks in this step include a timing analysis which resolves ambiguities, finds implicit functions and removes redundancies in the specification.

The third subprocess, implementation, uses structura function modules to implement the functions. The output of this step is a set of function modules and their interconnections. The goal of this step is to find a set of function modules which has a minimum cost, calculated by 
adding a different weight on each metric of the design. The calculation of cost of a design in this step is more complex than that in function abstraction. Metrics of a design include price, physical size, power consumption, speed and others that may be used to evaluate the final design. In certain implementations, such as using ICs in board level digital circuit design, using a spare complex module to implement a simpler function can be less costly than instantiating a new physical entity (IC). A function may be implemented by different types of function modules, a combination of several function modules or part of a function module. A function module may have several different physical structures with different emphasis on one or some metrics. There are libraries that store the implementation methods of functions and the available physical modules for a function module.

\section{Function abstraction from a behavioural} specification

Design optimisation is the key task in digital circuit design. This is especially true when the system specification is purely behavioural, since there are many different designs which can be made to implement a particular specification. The different designs come from different functions abstracted from the specification

The mapping from primitive operations to functions is many-to-many, i.e. different groups of primitive operations can be abstracted to the same function, and a group of primitive operations can be abstracted to several different functions. If part of the covered primitive operations of a function overlaps with the set of compiled primitive operations, this function may be selected to cover that portion of the desired behaviour. The remaining primitive operations of this selected function, having no overlap with the compiled set, then will be wasted. Hence, one of the design goals is to maximise the utilisation of each selected function.

Let us now use an example to illustrate the process of function abstraction. Suppose that we are to design a digital circuit to output four square-wave signais with the same waveform but different phases. Also, let each signal have a period of eight clock cycles. During each period, the signal should remain high for three clock cycles and then drop low. The time difference between two neighbouring signals is two clock cycles. The behavioural specification and the corresponding timing diagram is shown in Fig. 2.

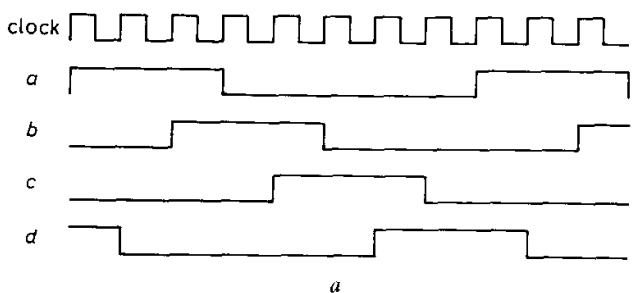

SIGNAL INPUT clk $=1$

OUTPUT $a, b, c, d=$

FUNCTION SYSTEM CLOCK $=\mathrm{clk}$

CLOCK $=a, b, c, d \quad$ PERIOD 8 DUTY CYCLE 3

EVENT $0=a$ DELAY 2 CLOCK CYCLES $b$

b DELAY 2 CLOCK CYCLES c DELAY 2 CLOCK CYCLES

Fig. 2 Digital circuit

$a$ Timing diagram

$b$ Description

238
After compilation, the behavioural specifications are translated into the following primitive operations:

Group 1:

(a) transmit(1, , $a, t A+i)$ for $i=0$ to 2 transmit $(0, a, t A+i)$ for $i=3$ to ?

(b) transmit $(1, b, t B+i)$ for $i=0$ to 2 transmit $(0, b, t B+i)$ for $i=3$ to 7

(c) transmit $(1,, c, t C+i)$ for $i=0$ to 2 transmit $(0, c, t C+i)$ for $i=3$ to 7

(d) transmit $(1, d, t D+i)$ for $i=0$ to 2 transmit $(0, d, t D+i)$ for $i=3$ to 7

Group 2:

(a) value $\left(a, v_{1}, t\right)$ transmit $\left(v_{1},, b, t+2\right)$

(b) value $\left(b, v_{2}, t\right)$ transmit $\left(v_{2},, c, t+2\right)$

(c) value $\left(c, v_{3}, t\right)$ transmit $\left(v_{3},, d, t+2\right)$

Group 1 comes from the clock declarations of the signals and Group 2 comes from the event descriptions. A square-wave signal comes from continuing to transmit 1 for certain cycles (duty cycle) and then transmitting 0 to it. Thus, three consecutive $1 \mathrm{~s}$, and then five consecutive $0 \mathrm{~s}$ are transmitted to signal $a, b, c$ and $d$ at different starting time instances $t A, t B, t C$ and $t D$. The delay relationship between two signals is interepreted as keeping the value of one signal for the delayed clock periods and then transmitting it to the other. Thus, value declarations of signal $a, b$ and $c$ are generated separately, and the values are transmitted to the desired signal, respectively.

The next step is to abstract primitive operations to functions. For this, several possibilities will be explored in detail. These operations can be covered by a pattern generation function which transmits fixed data to the outputs on the control of its control signals sequentially The covered operations of this function are

$$
\begin{aligned}
& \operatorname{transmit}\left(p t_{1 i}, c n t l_{i}, a,\right) \\
& \operatorname{transmit}\left(p t_{2 i}, c n t l_{i}, b,\right) \\
& \operatorname{transmit}\left(p t_{3 i}, c n t l_{i}, c,\right) \\
& \operatorname{transmit}\left(p t_{4 i}, c n t l_{i}, d,\right) \text { for } i=1 \text { to } 8
\end{aligned}
$$

To implement the set of compiled primitive operations, eight sets of 4-bit patterns $\left(p t_{11}-p t_{41}\right.$ through $\left.p t_{18}-p t_{48}\right)$ will be output to signals $a-d$ sequentially. One supporting operation is needed to generate the appropriate control signals

value(cntl $, 1, t+i-1)$

These operations can be covered by a function control
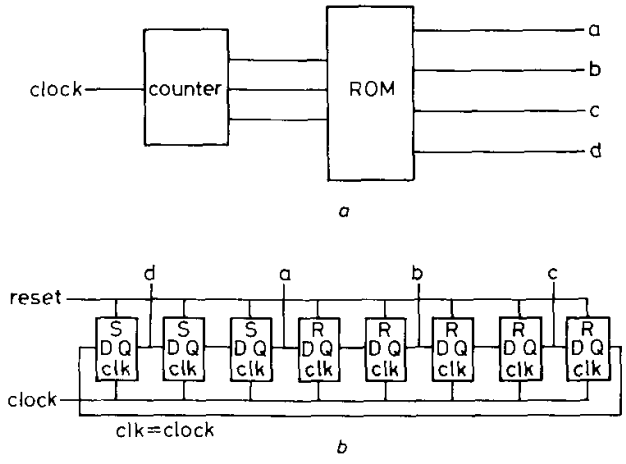

Fig. 3 Two designs caused by different functions abstracted

JEE PROCEEDINGS, Voi. 136, Pt. G, No. 5, OCTOBER 1989 
sequence generation which generates a pulse signal to eight signals sequentially. We can use a ROM (Read Only Memory) and a 3-bit counter to implement these functions. The circuit is shown in Fig. 3a.

The transmit operations in group 1(a) can also be covered by a function parallel-to-serial conversion with output $a$ whose behaviour is described in Section 3.1. We can also abstract functions parallel-to-serial conversion with output $b, c$ and $d$ from group $1(b)$ and $2(a), 1(c)$ and $2(b)$ and $1(d)$ and $2(c)$, respectively. The behaviour of the system clock, which is rising every clock cycle, can cover the second support operation. A function reset signal generation is needed to cover the first support operation. The structural implementation of these abstracted functions is shown in Fig. $3 b$.

\section{Formulation of the function abstraction process}

We have implemented the function abstraction subprocess (written in PASCAL on a VAX 11/780) [16] using heuristic best-first search strategy (A* algorithm) [17]. We envision the solution space as a huge search tree. There are four sets of behavioural elements in each node. They are sets of selected functions, uncored operations, added operations and covered operations. Going from the current node to a child node in this search tree results in the selection of a new function to cover some uncovered compiled primitive operations. The set of selected functions contains all the functions that have been selected so far to cover operations that were originally in the set of uncovered operations. The set of uncovered operations are those primitive operations that are not covered by the set of selected functions. The set of added operations are primitive operations covered by the set of selected functions but which did not appear in the set of uncovered operations i.e. they are not yet used. The set of covered operations are the operations which were in the set of uncovered operations but have since been covered by some selected function.

At the beginning of the search process, the root node of the search tree is initiated with all required primitive operations in its set of uncovered operations and all other sets empty. Then, iteration of node generation is performed until a solution using the best-first heuristic search is found. A node expansion is performed by finding all the functions that cover one or more operations in the uncovered set. For each of these functions, one child node is generated. The uncovered operations covered by the function are moved to the set of covered operations of the corresponding node. Unused operations that are covered by the added function are added to the set of added operations.

\subsection{Best first search strategy}

The goal of this search process is to select the minimum number of functions that will cover all the primitive operations which were uncovered in the initial state, with a minimum number of unused added operations. The search procedure is described as follows:

(i) Initialise the root node to contain the set of uncovered operations produced by behavioural specifications compilation. The other three sets are initially empty

(ii) Until a solution (a node with minimum cost and an empty set of uncovered operations) is found, repeat the following steps:

(a) Pick the best node (the one with the minimum distance to a solution, as estimated by $h^{\prime}$ ) and expand it. The

IEE PROCEEDINGS, Vol 136, Pt. G, No. 5, OCTOBER 1989 expansion of a node is to designate each operation from the set of uncovered operations in turn, and identify functions that can cover this operation. Each such pairing generates a potential child node. If the set of selected functions for a potential child node matches the set of selected functions from some previously generated node, that potential child node is not generated. Otherwise, copy the four sets (selected functions, added operations, covered operations and uncovered operations) to the child node. In the child node, add the newly selected function to the set of selected functions. Add those operations which that function covers to the set of added operations. Add the supporting operations for that function to the set of uncovered operations. Delete those operations which appear in both the set of uncovered operations and the set of added operations, and move the deleted operations to the set of covered operations

(b) Compute the cost of each new node according to the heuristic function $h$ ', which approximates node 'goodness'. One heuristic function approximates cost by summing the numbers of elements in thesets of selected functions, uncovered operations and added operations. Weights can be used to adjust the contribution of each such number to the computed cost.

The heuristic function $h^{\prime}$ is calculated as

$$
h^{\prime}=\text { weight } \times \text { number of uncovered operations }
$$

+ number of added operations + number of functions

Its goal is to have minimum numbers of functions and added operations. A larger weight on uncovered operations will result in using functions that cover more operations. More added (unused) operations are included but less processing time is needed in finding a solution.

\subsection{Planning and meta-planning techniques}

Planning is an artifical intelligence problem-solving technique. In planning, it is often assumed that the overall design goal is composed of a conjunction of interacting subgoals. The objective of planning then is to develop a 'plan' to achieve the overall goal in an orderly manner Meta-planning is concerned with managing these interactions to help determine the order in which decisions are made (the subgoal should be addressed next) and how each decision is resolved (the subplan should be implemented to achieve that subgoal). That is, metaplanning is concerned with the control of planning decisions, i.e. knowing when and how to make commitments to achieve a subgoal and when not to [18].

The simplest way of managing interactions is to determine the order in which subgoals could be achieved for the overall subgoal to be achieved [19]. This ordering is done to insure that the action of achieving one subgoal does not undo an already achieved one, or block an as yet unachieved one. In function abstraction, the equivalent meta-planning action would be to order the sequence in which primitive operations are to be covered Although this type of meta-planning could be sensitive to critical subgoals, putting them at the head of the sequence, it does not provide any guidance in determining which subplan (function) should be selected to achieve that subgoal in a manner sensitive to the remaining unachieved subgoals.

Our meta-planning strategy is based on the common sense that the function is selected to tightly cover the largest number of uncovered primitive operations, i.e. introducing the least number of added primitive oper- 
ations, which are also covered by the function but do not cover any uncovered primitive operations.

\subsection{Node expansion strategies}

During the function search subprocess, for each primitive operation in the set of uncovered operations we need to find functions that cover the operation. One child node is generated for each found function. The child node adds the found function to its function set and moves those operations the function covers to the set of covered operations. An operation often can be covered by several functions, especially for the sequential operation store.

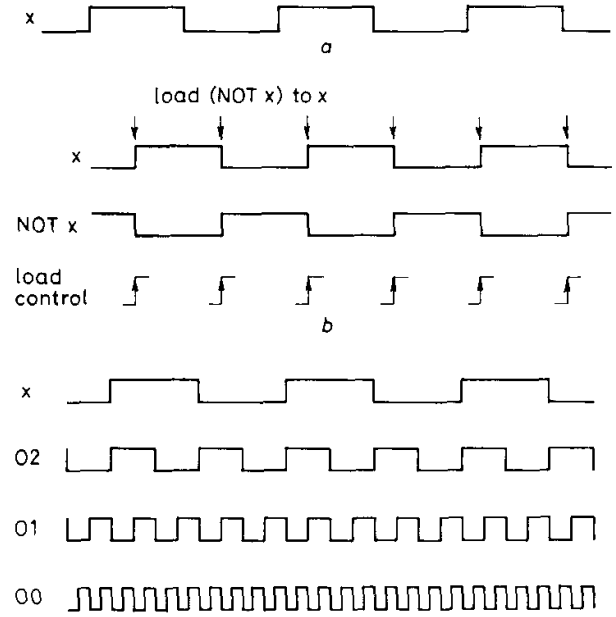

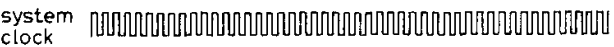
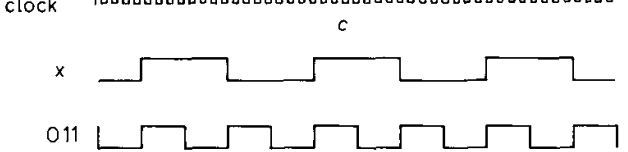

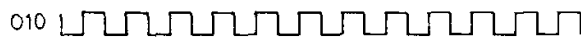

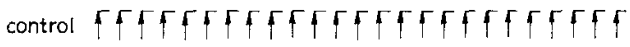
$d$
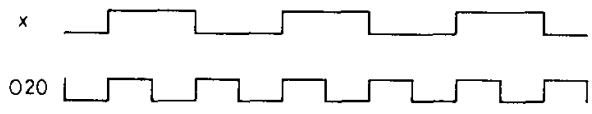

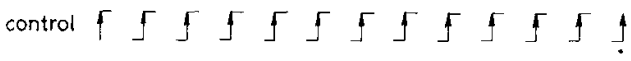

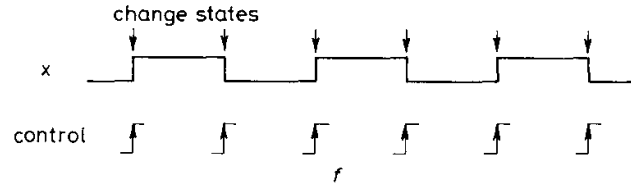

Fig. 4 Behaviour of functions

Each of which covers the store $(x-,, x, 8$ r) operation

a Desired behaviour

$a$ Beshaviour of a load function and its support operation

c Behaviour of a 4 -bit count function

d Behaviour of a 3-bit count function

$e$ Behaviour of a 2-bit count function

$f$ Behaviour of a change states function
For example, a store $(x-,, x, 8 r)$ operation, whose behaviour is shown in Fig. $4 a$, may be covered by following functions:

(i) A load function with support operation $\operatorname{NOT}(x$,

$x-$,$) and the value of a load control signal. The behav-$ iour of these logical entities is shown in Fig. $4 b$

(ii) A 4-bit count function with a system clock as its clock and unused operations store $\left(\mathrm{O}_{0}-,, \mathrm{O}_{0}, 1 r\right)$ store $\left(\mathrm{O}_{1-},, \mathrm{O}_{1}, 2 r\right)$ and store $\left(\mathrm{O}_{2-},, \mathrm{O}_{2}, 4 r\right)$ for the lower order output bits. The behaviour of a 4-bit count function is shown in Fig. $4 c$

(iii) A 3-bit count function with $2 r$ (a signal which changes its states every two clock cycles) as its clock and unused operations store $\left(\mathrm{O}_{10^{-}},, \mathrm{O}_{10^{-}}, 2 r\right)$ and store $\left(\mathrm{O}_{11^{-}}\right.$, $\left.\mathrm{O}_{11}, 4 r\right)$ for the lower order-output bits. The behaviour of a 3-bit count function and its control signal is shown in Fig. $4 d$

(iv) A 2-bit count function with $4 r$ as its clock and unused operations store $\left(\mathrm{O}_{20},, \mathrm{O}_{20}, 4 r\right)$ for the lower order output bit. The behaviour of a 2 -bit count function and its control signal is shown in Fig. $4 e$

(v) A change states function of $x$ every eight clock cycles repeatedly. Its behaviour is shown in Fig. $4 f$.

We have two substantially different node expansion strategies for the $A^{*}$ algorithm in the program. One is generating all possible child nodes. For example, while expanding a node which contains the operation store $(x-$, $x, 8 r$, all the child nodes that contain the above functions should be generated. Fig. 5 illustrates the generation of the first two child nodes in the node expansion step. The second node expansion strategy generates only one child node for each type of function. The types of function that cover the above operation are load, change states and count. There is one function for function type load and change states, and several functions for function type count. We choose the most promising one from each type of these functions. Thus, if we have $m$ possible functions which can be categorized into $n$ types, we will have $n$ branches from the node, one for each of the types. The most promising function is the one that contains at most one unused operation between two operations. For example, the above operation can be covered by a 4-bit count function only if there is an operation store $(b-,, b$, $2 r$ ) (the second lowest bit). There is an unused operation (the third lowest bit) of the function between these two operations and another unused operation which is the lowest bit (the bit between the second lowest and nothing) of the count function.

The performance of the best-first search strategy should be compared with some other blind search strategies to show their superiority. Comparison with the depth-first search strategy is meaningless. Since for every uncovered primitive operation, there is a function that

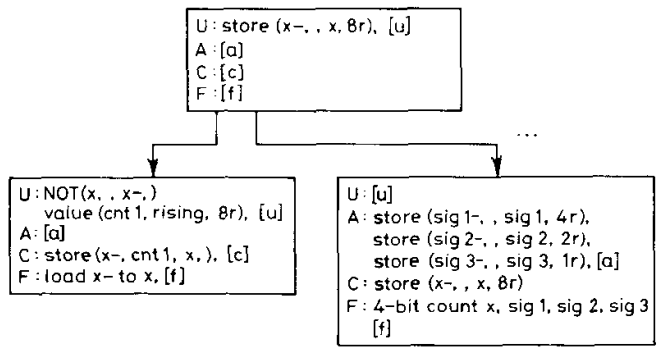

Fig. 5 Example of node expansion

IEE PROCEEDINGS, VOL I36, Pt. G, NO S, OCTOBER 1989 
covers it, applying the depth-first search strategy can always find a solution without backtracking. The solution will be trivial functions, where each of them covers only one primitive operation or large functions that include too many unused operations. Which one of these extremely nonoptimum situations occurs depends on the order of selecting functions. We thus select the breadthfirst search stragey for comparison and to show the superiority of our methods. All possible child nodes are expanded in each node expansion.

\subsection{Timing analysis of primitive operations}

While expanding a node, domain specific knowledge is employed to determine whether some of the uncovered operations can be covered by a function. The domain knowledge comes from two sources. The first one is the knowledge explicitly encoded in the representation of the function, i.e. certain primitive operations may match (with unification of variables) some covered operations in a function. The second one is the knowledge of performing timing analysis to match uncovered operations with those operations covered by a function. Here, timing analysis is defined as the task of exploring relationships among primitive operations. These relationships could include timing, input, output or others, e.g. the input/ output relationship of the change states function or the waveform regularity of the count function. Search for similarity is needed to accomplish this task.

Below, we give a detailed account on how to exploit the timing relationships among a set of unrepeated store primitive operations to see if they can be covered by a count function.

A count function is composed of several repeated change states functions; each of them may be represented by two primitive operations, namely store $(a, c, b, t)$ and $\operatorname{NOT}(b, a, a)$ On the other hand, each change states function may also be represented by a single operation store $(b-, c, b, t)$, where $b$-denotes the complement of $b$. If a signal changes its states every $P$ clock cycles, and $P$ is a power of 2, then the signal may be realised as one of the output bits in a count function. Furthermore, even if the timing parameter is a constant, it is still possible that operation will be repeated after certain intervals. Timing analysis is needed to find when such a repetition begins and ends. Then the timing parameter will take the format of

$$
c_{1}+c_{2} r
$$

This indicates that the selected count function should be reset at time $c_{1}$, and then repeated every $c_{2}$ clock cycles. This, in turn, requires the use of supporting primitive operations to reset the count function and transfer the count output to the designated signal during the desired time interval. The behaviour of the count function and support operations are shown in Fig. 6.

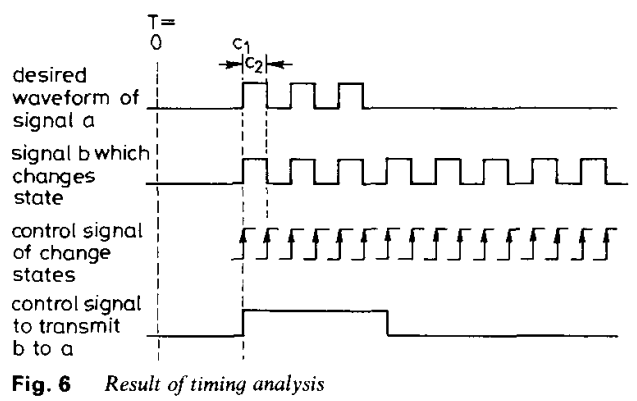

IEE PROCEEDINGS, Vol. 136, Pt. G, No. 5, OCTOBER 1989

\section{Examples}

In this Section, we use a number of design examples to demonstrate the function abstraction subprocess and to compare the performance of the breadth-first search and the best-first search strategies.

\subsection{Design examples}

The first set of primitive operations to which the various search methods will be applied for comparison purposes are

$$
\begin{aligned}
& \text { store }(a-,, a, \mathbf{1 6} r) \\
& \text { store }(b-,, b, \mathbf{8 r}) \\
& \text { store }(c-,, c, \mathbf{2 r}) \\
& \operatorname{AND}(a, b, c,, d)
\end{aligned}
$$

Its timing diagram is shown in Fig. 7. The functions selected by the three-node expansion strategies are identical, i.e. a 5-bit count and an and function. The statistics of performance of each searching strategy are shown in Table 1, where the weight is that of uncovered operations. Method 1 uses the $\mathrm{A}^{*}$ algorithm and expands all possible child nodes. Method 2 uses the $A^{*}$ algorithm but expands only the most promising child nodes. Method 3 uses breadth-first search to find the first solution. In this table a hyphen is used to indicate that the program cannot find the result in reasonable amount of time and the job has to be aborted. The execution time is in ms.

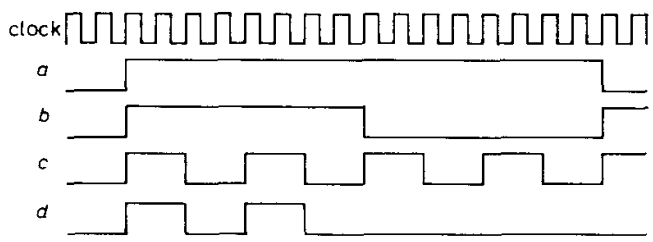

Fig. 7 Timing diagram

Table 1 :

\begin{tabular}{lllllll}
\hline & \multicolumn{3}{c}{ Weight $=1$} & \multicolumn{4}{c}{ Weight $=2$} \\
\hline Method & $\begin{array}{l}\text { Number of } \\
\text { expansion }\end{array}$ & $\begin{array}{l}\text { Expanded } \\
\text { node }\end{array}$ & Time & $\begin{array}{l}\text { Number of } \\
\text { expansion }\end{array}$ & $\begin{array}{l}\text { Expanded } \\
\text { node }\end{array}$ & Time \\
\hline A* & 4 & 43 & 600 & 2 & 21 & 317 \\
$\begin{array}{l}\text { A*, Meta- } \\
\text { planning }\end{array}$ & 5 & 27 & 384 & 3 & 15 & 250 \\
$\begin{array}{l}\text { Breadth- } \\
\text { first }\end{array}$ & 4 & 45 & 750 & - & - & - \\
\hline
\end{tabular}

Next, the order of the above primitive operations is permuted as follows:

$$
\begin{aligned}
& \text { store }(c-,, c, \mathbf{2 r}) \\
& \text { store }(b-,, b, \mathbf{8} \boldsymbol{r}) \\
& \text { store }(a-,, a, \mathbf{1 6 r}) \\
& \operatorname{AND}(a, b, c, d,)
\end{aligned}
$$

\begin{tabular}{|c|c|c|c|c|c|c|}
\hline \multirow[b]{2}{*}{ Method } & \multicolumn{3}{|c|}{ Weight $=1$} & \multicolumn{3}{|c|}{ Weight $=2$} \\
\hline & $\begin{array}{l}\text { Number of } \\
\text { expansion }\end{array}$ & $\begin{array}{l}\text { Expanded } \\
\text { nodes }\end{array}$ & Time & $\begin{array}{l}\text { Number of } \\
\text { expansion }\end{array}$ & $\begin{array}{l}\text { Expanded } \\
\text { nodes }\end{array}$ & Time \\
\hline $\mathrm{A}^{*}$ & 4 & 43 & 600 & 2 & 21 & 300 \\
\hline $\begin{array}{l}\text { A*, Meta- } \\
\text { planning }\end{array}$ & 5 & 27 & 400 & 3 & 15 & 250 \\
\hline $\begin{array}{l}\text { Breadth- } \\
\text { first }\end{array}$ & 14 & 184 & 4650 & & - & . \\
\hline
\end{tabular}

Although different search strategies still arrived at the same set of functions, the performance statistics, as listed in Table 2, are different.

Table 2: 
The second experiment uses the set of primitive operations of a sequence detector, which outputs a 1 to the output signal out, whereas the binary data stream from input signal $x$ is $\mathbf{1 0 1 1}$. The set of primitive operations are

value $\left(x, v_{1}, t_{1}\right)$

value $\left(x, v_{2}, t_{1}+1\right)$

value $\left(x, v_{3}, t_{1}+2\right)$

$\operatorname{value}\left(x, v_{4}, t_{1}+3\right)$

$\operatorname{NOT}\left(v_{2}, v_{5},\right)$

$\operatorname{AND}\left(v_{1}, v_{5}, v_{3}, v_{4}\right.$, out, $\left.t_{1}+3\right)$

When we assign 1 to the weight for uncovered operations, the functions serial-to-parallel conversion, started at time $t_{1}$, not, and and control signal generation, for starting the serial-to-parallel conversion function, will be selected. If the weight is set to 2 , a different set of the functions loads, of signal $x$ at time $t_{1}, t_{1}+1, t_{1}+2$ and $t_{1}+3$, and, not and control signal generation, for those load functions, will be selected. The statistics are compiled in Table 3. In this example, the computation times of the two-node expansion method are comparable. This is because there is no store operation in the given set of primitive operations. Otherwise, there will be many different instances of count function which may be selected. The difference in time of these two-node expansion methods is caused by the introduction of some store operations as support operations for selected functions. The breadth-first search strategy cannot find a solution in reasonable time before running out of computer memory (6 Mbytes allocated). This is because too many child nodes have to be generated to cover those transmit operations. In this case, permuting the order sequence of primitive operations does not improve the performance.

Table 3:

\begin{tabular}{lllllll}
\hline & \multicolumn{3}{c}{ Weight $=1$} & \multicolumn{3}{c}{ Weight $=2$} \\
\hline Method & $\begin{array}{l}\text { Number of } \\
\text { expansion }\end{array}$ & $\begin{array}{l}\text { Expanded } \\
\text { node }\end{array}$ & Time & $\begin{array}{l}\text { Number of } \\
\text { expansion }\end{array}$ & $\begin{array}{l}\text { Expanded } \\
\text { node }\end{array}$ & Time \\
\hline $\begin{array}{l}\text { A }^{*} \\
\begin{array}{l}A^{*}, \text { Meta- } \\
\text { planning }\end{array}\end{array}$ & 295 & 1780 & $\mathbf{1 6 1 7 1 7}$ & $\mathbf{1 0}$ & 57 & 1033 \\
$\begin{array}{l}\text { Breadth- } \\
\text { first }\end{array}$ & - & 1450 & 110317 & 10 & 57 & 116 \\
\hline
\end{tabular}

\subsection{Comparison of search strategies}

The function abstraction program has been tested using 21 sets of compiled primitive operations to compare different search strategies. The results are compiled and listed in Tables 4 to 8 . Based on these tables, the following observations can be made:

(i) The number of functions selected seems to be independent of the number of compiled primitive operations. Instead, it depends more on the relevance among the set of primitive operations. If the primitive operations are closely related, usually fewer functions will be selected. For example, there are only three primitive operations in case 2

$\operatorname{store}\left(a_{-},, a, t_{1}\right)$

value $\left(c\right.$, rising, $\left.t_{2}\right)$

$\operatorname{NOT}\left(a, s g A B, t_{2}\right)$

Because these primitive operations are relatively independent, each requires a separate function to cover. In particular, the functions selected are change states, control signal generation at time $t_{1}$, control signal generation at time $t_{2}$ and not. The function control signal generation at time $t_{1}$ is used to cover the support operation of function change states.
Table 4: Numbers of initial primitive operations and found functions

\begin{tabular}{lllllll}
\hline & $\begin{array}{l}\text { Number } \\
\text { of ops }\end{array}$ & \multicolumn{2}{l}{\begin{tabular}{l} 
A* algorithm \\
\cline { 2 - 5 } 1**
\end{tabular}} & \multicolumn{2}{l}{ Meta-planning } & $\begin{array}{l}\text { Breadth-first } \\
\text { search }\end{array}$ \\
\hline 1 & 8 & 3 & 3 & 5 & 5 & 3 \\
2 & 3 & 4 & 4 & 4 & 4 & - \\
3 & 12 & 6 & 6 & 6 & 6 & - \\
4 & 8 & - & - & - & 13 & - \\
5 & 27 & 3 & 3 & 3 & 3 & 2 \\
6 & 6 & 1 & 1 & 1 & 1 & 1 \\
7 & 4 & 3 & 3 & 4 & 4 & 1 \\
8 & 3 & 3 & 4 & 3 & 4 & 2 \\
9 & 18 & 3 & 3 & 3 & 3 & - \\
10 & 14 & 1 & 1 & 1 & 1 & 1 \\
11 & 8 & 5 & 2 & 5 & 2 & - \\
12 & 24 & 1 & 1 & 1 & 1 & - \\
13 & 12 & 1 & 1 & 1 & 1 & 1 \\
14 & 12 & 1 & 1 & 1 & 1 & 1 \\
15 & 4 & 4 & 4 & 4 & 4 & 3 \\
16 & 4 & 4 & 4 & 4 & 4 & 3 \\
17 & 6 & 1 & 1 & 3 & 3 & 1 \\
18 & 6 & 1 & 1 & 3 & 3 & 1 \\
19 & 14 & 7 & 4 & 7 & 4 & - \\
20 & 7 & - & - & - & 14 & - \\
21 & 18 & 4 & 4 & 4 & 4 & - \\
\hline
\end{tabular}

** Weight of uncovered operations

Table 5 : Numbers of added operations

\begin{tabular}{llllll}
\hline & \multicolumn{2}{l}{ A* algorithm } & \multicolumn{2}{c}{ Meta-planning } & $\begin{array}{l}\text { Breadth- } \\
\text { first }\end{array}$ \\
\hline & 1 & 2 & 1 & 2 & 0 \\
1 & 0 & 0 & 0 & 0 & - \\
2 & 0 & 0 & 0 & 0 & - \\
3 & 0 & 0 & 0 & 0 & 27 \\
4 & - & - & - & 0 & 2 \\
5 & 0 & 4 & 0 & 4 & 6 \\
6 & 2 & 2 & 2 & 2 & 4 \\
7 & 2 & 3 & 2 & 2 & - \\
8 & 0 & 0 & 0 & 0 & 0 \\
9 & 0 & 0 & 0 & 0 & - \\
10 & 0 & 0 & 0 & 0 & - \\
11 & 0 & 4 & 0 & 4 & 0 \\
12 & 0 & 0 & 0 & 0 & 2 \\
13 & 0 & 0 & 0 & 0 & 2 \\
14 & 0 & 0 & 0 & 0 & 2 \\
15 & 0 & 0 & 0 & 0 & - \\
16 & 0 & 0 & 0 & 0 & - \\
17 & 2 & 2 & 0 & 0 & - \\
18 & 2 & 2 & 0 & 0 & 2 \\
19 & 0 & 4 & 0 & 4 & - \\
20 & - & - & - & 0 & 0 \\
21 & 0 & 0 & 0 & 0 & \\
\hline
\end{tabular}

Table 6 : Numbers of nodes expanded

\begin{tabular}{llllll}
\hline & \multicolumn{3}{l}{ A* } & algorithm & \multicolumn{2}{l}{ Meta-planning } & $\begin{array}{l}\text { Breadth- } \\
\text { first }\end{array}$ \\
\cline { 2 - 6 } & 1 & 2 & 1 & 2 & 205 \\
1 & 3 & 3 & 7 & 5 & - \\
2 & 6 & 4 & 6 & 4 & - \\
3 & 6 & 6 & 6 & 6 & - \\
4 & - & - & - & 13 & 765 \\
5 & 5 & 4 & 5 & 4 & 1 \\
6 & 1 & 1 & 1 & 1 & 1 \\
7 & 165 & 3 & 62 & 4 & 3 \\
8 & 5 & 4 & 5 & 4 & - \\
9 & 3 & 3 & 3 & 3 & 1 \\
10 & 1 & 1 & 1 & 1 & - \\
11 & 5 & 2 & 5 & 2 & - \\
12 & 2 & 2 & 2 & 2 & 1 \\
13 & 1 & 1 & 1 & 1 & 1 \\
14 & 1 & 1 & 1 & 1 & 13 \\
15 & 4 & 4 & 4 & 4 & 1 \\
16 & 4 & 4 & 4 & 4 & 1 \\
17 & 1 & 1 & 4 & 4 & - \\
18 & 1 & 1 & 4 & 4 & - \\
19 & 7 & 4 & 7 & 4 & - \\
20 & - & - & - & 14 & 5 \\
21 & 5 & 5 & 5 & 5 & \\
\hline
\end{tabular}

IEE PROCEEDINGS, Vol. 136, Pt. G, No. 5, OCTOBER 1989 
Table 7: Numbers of generated nodes

\begin{tabular}{|c|c|c|c|c|c|}
\hline & \multicolumn{2}{|c|}{$\mathrm{A}^{*}$ algorithm } & \multicolumn{2}{|c|}{ Meta-planning } & \multirow{2}{*}{$\begin{array}{l}\text { Breadth- } \\
\text { first }\end{array}$} \\
\hline & 1 & 2 & 1 & 2 & \\
\hline 1 & 55 & 55 & 47 & 39 & 5465 \\
\hline 2 & 15 & 10 & 15 & 10 & - \\
\hline 3 & 48 & 48 & 48 & 48 & - \\
\hline 4 & - & - & - & 122 & - \\
\hline 5 & 55 & 41 & 55 & 41 & 4411 \\
\hline 6 & 7 & 7 & 7 & 7 & 2 \\
\hline 7 & 2891 & 55 & 334 & 22 & 13 \\
\hline 8 & 23 & 17 & 23 & 17 & 18 \\
\hline 9 & 37 & 37 & 37 & 37 & - \\
\hline 10 & 24 & 24 & 24 & 24 & 18 \\
\hline 11 & 43 & 25 & 43 & 25 & - \\
\hline 12 & 60 & 60 & 60 & 60 & - \\
\hline 13 & 20 & 20 & 20 & 20 & 10 \\
\hline 14 & 20 & 20 & 20 & 20 & 20 \\
\hline 15 & 21 & 21 & 21 & 21 & 87 \\
\hline 16 & 27 & 27 & 27 & 27 & 122 \\
\hline 17 & 37 & 37 & 24 & 24 & 3 \\
\hline 18 & 37 & 37 & 24 & 24 & 12 \\
\hline 19 & 82 & 55 & 82 & 55 & . \\
\hline 20 & - & 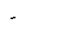 & - & 121 & - \\
\hline 21 & 56 & 56 & 56 & 56 & - \\
\hline
\end{tabular}

Table 8: Execution time

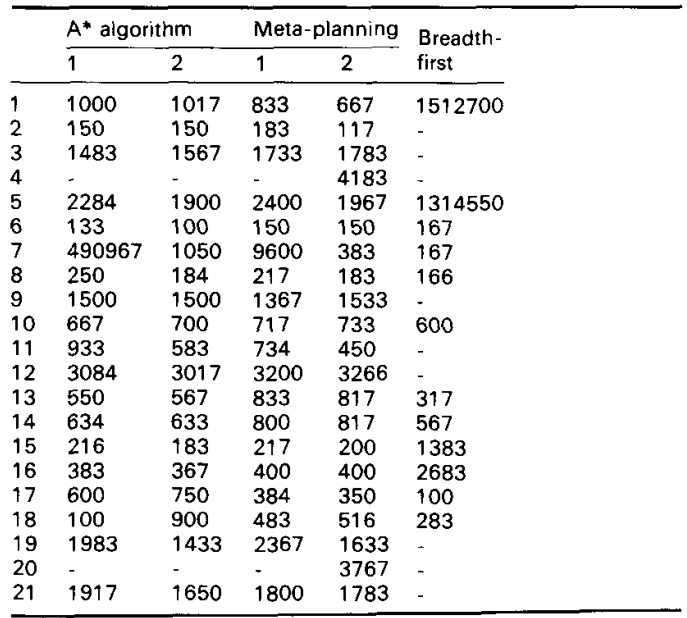

As another example, there are 24 primitive operations in total in case 12

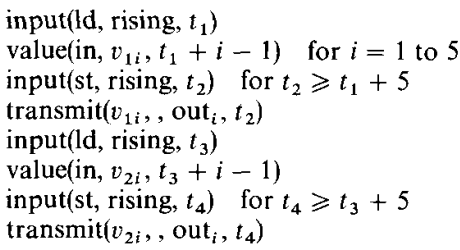

However, since these primitive operations are highly related, one function only, a 5-bit serial-to-parallel conversion function, will be sufficient to cover all these primitive operations

(ii) The breadth-first search is computationally too costly to be a practical search strategy. In our experiments, whenever there are more than four functions to be selected, the breadth-first search process had to be aborted due to excessive usage of CPU time. Instead, the planning technique seems to have better search efficiency in cases where more sequential operations are needed, such as cases 4, 7, 17, 18 and 20 . Note that in cases 4 and
20 , it helped solve the problems which even the $A^{*}$ algorithm failed to do. For example, in case 4 , the set of primitive operations are

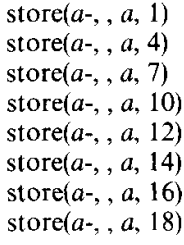

A timing analysis is carried out to explore functions which implicitly cover this set of primitive operations. The only results found are three change states functions at time, 1, 4 and 7, a 4-bit count function started at time 10 and stopped at time 18, a gate function which transmits outputs of the count function to signal $a$, a set function and a reset function of a signal which enables operations of the count function, control signal generation functions at time $1,4,7,10$ and 18 and a clock of the count function

(iii) Increasing the weight of uncovered primitive operations usually cuts the execution time. It also frequently results in a fewer number of functions being selected, as it favours the use of functions that cover more primitive operations. But these benefits come at a price: a larger weight of uncovered operations discourages the search direction from backtracking to a higher level node in the search tree where a better solution may reside. In other words, using larger weights on the uncovered functions is a greedy heuristic which improves the search efficiency at the risk of sticking in a local minimum, and therefore missing the globally optimised solution. For example, in case 8 , where three primitive operations are given

$\operatorname{transmit}\left(c,, d, t_{1}\right)$

transmit $\left(b, d, t_{1}+1\right)$

Value $\left(a, c, t_{1}\right)$

If the weight of uncovered operations is set to 1 , two gate functions and a control signal generation function will be selected. However, if it is set to 2, one more load function will be selected which clearly is an inferior solution

(iv) In several cases, such as 6, 7, 13, 14, 17 and 18, the breadth-first search strategy arrived at solutions faster than other methods. This is because, in these cases, only one function is selected. With the breadth-first search strategy, the process of node expansion will be halted once the first feasible solution is found. Clearly, this may result in inferior solutions. On the other hand, with other methods, the cost of all unexpanded nodes will be compared after node expansion. At the cost of a few more node expansion iterations, often a better solution can be obtained. For example, the set of primitive operations in case 7 are

$$
\begin{aligned}
& \text { store }(b-,, b, 2 r) \\
& \text { store }(e-,, e, 8 r) \\
& \text { store }(d-,, d, 128 r) \\
& \text { store }(f-,, f, 512 r)
\end{aligned}
$$

Using breadth-first search, a 10-bit count function is found to cover these operations. Using the $A^{*}$ algorithm, the result contains a 3 -bit count function controlled by the system clock and a 2-bit count function, together with a control signal generation function. Using the planning technique, the result includes two 2-bit count functions and two control signal generation functions 
Although the number of functions selected is larger using the latter two methods, they include less unused primitive operations and may lead to a more economical design.

(v) The performance of the breadth-first search is very much dependent on the order in which the set of primitive operations is arranged. To see this point, the test data sets 13 and 14,15 and 16 , as well as 17 and 18 , are, respectively, three pairs of data sets; each has the same set of primitive operations but with different orders. It turns out that the execution time using the breadth-first search has much larger variations than that of using the other two methods.

For example, the set of primitive operations in both cases 15 and 16 are

$$
\begin{aligned}
& \operatorname{transmit}\left(b, c_{1}, c\right) \\
& \operatorname{transmit}\left(b, c_{2}, e,\right) \\
& \operatorname{AND}\left(a_{1}, a_{2}, c_{1},\right) \\
& \operatorname{AND}\left(a_{1}, a_{2}-, c_{2},\right)
\end{aligned}
$$

Using the breadth-first search method, it takes $1383 \mathrm{~ms}$ to compute case 15 which yields two and functions and a 1-line-to-4-line demultiplex function, and $2683 \mathrm{~ms}$ to compute case 16 which yields the same set of functions. On the other hand, with the two other methods, the differences are not that significant; two gate functions and two and functions are selected

(vi) In some cases, the number of node expansions using the planning method may be greater than that of the A* methods. Nevertheless, the number of generated nodes of the planning method is always less than or equal to that of $A^{*}$ methods. This is because the planning method prunes the more unfeasible nodes during the search process.

\section{Conclusion}

We have reported the result of using heuristic search and a simple meta-planning technique of artificial intelligence in a minimisation problem - function abstraction of digital circuit design. There are two minimisation subprocesses in our automatic design system. The first one is function abstraction which finds the functions the target system performs. The other one is structure implementation which realises the functions by structural modules. Adding the function abstraction subprocess is a distinguished feature of the system. It finds the essence of the target behaviour rather than binds functions in the specification directly to function modules. This provides more opportunity for performing minimisation. We have coded this subprocess using PASCAL on VAX 11/780. Experimental results have shown the heuristic search efficiently reduces the huge design space. Adding metaplanning the search process usually leads to the same solution but in a shorter time.

We are designing better heuristic functions and more elaborated meta-planning techniques in the minimisation step as an effort to get better designs in a shorter time. We are also applying the heuristic search and meta- planning techniques on the other minimisation step structure implementation. The interface between these two subprocesses to resolve their interaction is also an important topic

\section{Acknowledgment}

Y.H. Hu is supported by the National Science Foundation under contract MIP-8896111.

\section{References}

1 MCCLUSKEY, E.J. Jr.: 'Minimization of Boolean functions', Bell Syst. Tech. J., 1956, 35, (6), pp. 1417-1444

2 BRAYTON, R.K., HACHTEL, G.D., MCMULLEN, C.T., and SANGIOVANNI-VINCENTELLI, A.L.: 'Logic minimization algorithms for VLSI synthesis' (K luwer Academic Publishers, 1984)

3 HONG, S.J., CAIN, R.G., and OSTAPKO, D.L.: 'MINI: a heuristic HONG, S.J., CAIN, R.G., and OSTAPKO, D.L.: 'MINI: a heuristic
approach for logic minimization', IBM J. Res. Dev., 1974, 18, (5), pp. approach

4 DARRINGER, J., BRAND, D., GERBI, J.V., JOYNER, W.H. Jr. and TREVILLYAN, L.: "LSS: a system for production logic synthesis', IBM J. Res. Dev., 1984, 28, (5), pp. 537-545

5 DE GEUS, AJ., and COHEN, w: 'A rule-based system for optimizing combinational logic', IEEE Des. Test Comput., 1985, 2, (4), pp. 22-32

6 KOWALSKI, T.J.: 'An artificial intelligence approach to VLSI design' (Kluwer Academic Publisher, 1985)

7 KOWALSKI, T.J., and THOMAS, D.E.: 'The VLSI design automation assistant: an IBM system/370 design', IEEE Des. Test Comput., $1984,1,(1)$, pp. $60-69$

8 GRANACKI, J., KNAPP, D., and PARKER, A.: 'The ADAM advanced design automation system: overview, planner and natural language interface'. Proceedings of 22nd Design Automation Conference, Las Vegas, NV, USA, June 1985, pp. 727-730

9 KNAPP, D.W., and PARKER, A.C.: 'A design utility manager: the ADAM planning engine'. Proceedings of 23 rd Design Automation ADAM planning engine'. Proceedings of 23 rd Design
Conference, Las Vegas, NV, USA. June 1986, pp. 48-54

10 MITCHELL, T.M., STEINBERG, L.I., and SHULMAN, J.S.: 'A knowledge-based approach to design', IEEE Trans., 1985, PAMI-7, (5), pp. 502-510

11 BREWER, F.D., and GAJSKI, D.D.: 'An expert-system paradigm for design'. Proceedings of 23rd Design Automation Conference, Las Vegas, NV, USA, June 1986, pp. 62-68

12 SUBRAHMANYAM, P.A.: 'Synapse: an expert system for VLSI design', IEEE Trans. Comput., 1986, 19, (7), pp. 78-88

13 WU, J.G. HO, W.P.C. HU, Y.H., YUN, D.Y.Y., and PARNG, T.M.: 'A model-based expert system for digital systems design', Proc. Soc. Photo-Opt. Instrum. Eng., (US A), Applications of Artificial Intelligence $V$, May 1987,786 , pp. 12-19

14 MCFARLAND, M.C.: 'Using bottom-up design techniques in the synthesis of digital hardware from abstract behavioral descriptions'. Proceedings of 23rd Design Automation Conference Las Vegas, NV, USA, June, 1986, pp. 474-480

15 WU, J-G. HO, W P.C HU, YH YUN D.Y Y PARNG, T.M. and HSU, C.C.: "Multiple representations for automatic design of digital systems. International Symposium on VLSI Technology, Systems and Applications, Taipei, Taiwan, ROC, May 1987, pp. $124-128$

16 WU, J.-G., HO, W.P.C., HU, Y.H., YUN, D.Y.Y., and YU, H.J 'Function search from behavioral description of a digital system' Proceedings of 24th Design Automation Conference, Miami, FL, USA, June 1987, pp. 574-579

17 RICH, E.: 'Artificial Intelligence' (McGraw-Hill, 1983)

18 STEFIK. M.: 'Planning and meta-planning (MOLGEN: part 2)', Artif. Intell., 1981, 16, (2), pp. 141-169

19 SACERDOTI, E.D.: 'The nonlinear nature of plans'. Proceedings of the 4th International Joint Conference on Artificial Intelligence, 1975, pp. 206-214 